\title{
Programming simulations of cognitive processes: An example of building macrostructures
}

\author{
SHERYL R. YOUNG \\ Carnegie Group, Inc., Pittsburgh, Pennsylvania
}

\begin{abstract}
Information is provided on the process of creating psychological simulations using LISP. A simulation is described, and the difference between progamming and simulation and "normal" programming is also provided, along with programming and debugging tips.
\end{abstract}

The purpose of this presentation is to provide insight into the process of creating psychological simulations using LISP. ${ }^{1}$ It is assumed that the reader knows how to program and has background in the process he/she would like to model, but does not have experience creating simulations. Therefore, a description of a simulation is provided along with a discussion of the difference between programming a simulation and "normal" programming. Then a sample simulation is described, followed by a procedural description of the processes involved in creating the sample simulation. Finally, programming and debugging tips are presented.

\section{SIMULATIONS: WHAT ARE THEY?}

A cognitive simulation is a program that emulates psychological processes, structures, and their interaction. Building a simulation is different from "normal" programming because, by nature, simulating is a theory refinement process, and therefore cyclical or iterative. Human mental processes tend to be ill-defined, and psychological theories are usually vague. By engaging in the simulation process, one is forced to refine the theory and eliminate fuzzy concepts. In other words, the programmer must precisely describe processes, structures, and their interaction, thereby refining the theory.

Specifically, simulation programming differs from "normal" programming in three important ways: the amount of prespecification performed prior to coding, the use of a psychologically valid code, and the process of cyclically coding.

\section{Prespecification}

The first difference between simulation programming and normal programming is the amount of prespecification of variables, functions, structures, and processes required prior to actual coding. Total prespecification is impossible because simulating is a gradual theory refinement process. When one creates a program to perform a statisti-

The simulation described in this manuscript was conducted as a portion of the author's doctoral dissertation at the University of Colorado. Reprint requests should be sent to Sheryl R. Young, c/o Carnegie Group, Inc., 650 Commerce Court, Pittsburgh, PA 15219. cal analysis (e.g., an analysis of variance), all required variables and procedures can be predetermined. It is easy to prespecify the types of data structures needed and the total number of variables required. If one uses subroutines or functions, one can also tell what each will do, all before writing the first line of code. However, when programming a simulation, the programmer knows none of these things in their entirety in advance. Although an experienced simulator can prespecify more and more of these items, no one can ever know it all. In LISP simulations, variables are created in both progs ${ }^{2}$ and functions as they are needed. Functions are also created as they are needed and because they lead to simplifying portions of the code. Since the simulation process is a gradual, online, interactive refinement of a theory, the programmer never knows in advance exactly what is required, except for bare minimums, for example, short-term memory (STM), long-term memory (LTM), various sets of rules, etc. For example, in a simulation requiring the contents of STM to invoke production rules, one knows in advance that STM, its contents, and the production rules will need to be represented. But, how do the contents of STM trigger production rules? How many rules are fired at once? Does the number vary? If so, under what circumstances? What if there are two different rules that could fire? Should they both fire? Should you assess their relations to common goals and then decide? And so forth. These are just a few examples of the issues one faces.

\section{Psychological Validity}

The second major difference between simulating and programming in a standard context is that the code's psychological validity is a major concern. For example, the structural representation of STM in the code should match the structural representation assumed in cognitive theories. In regular programming, one is often concerned with using efficient data structures, procedures, and search techniques. In creating an initial simulation, efficiency is not an important consideration, unless you are creating a product for commercial purposes or an interactive type of program. Rather, it is very important to employ psychologically valid search techniques, data structures, and procedures. Unfortunately, this is a very difficult problem. The reason is that when programming, the investigator 
discovers many components and processes that are not addressed in the theory. Sometimes there is clear-cut experimental evidence suggesting how humans do whatever is in question. More frequently, however, there is either conflicting evidence or no evidence at all. In these cases, a best guess must be made about what people actually do. Such guesses can usually be experimentally evaluated, and the code can be altered if the guess turns out to be incorrect. The main point is that one program in a psychologically valid possibility.

\section{The Cyclical Nature of Theory Refinement}

Earlier, I stated that one way in which simulation programming differs from normal programming is that prespecification of all components and processes is impossible. Because all possibilities cannot be specified, the third manner in which simulation programming differs from normal programming is that simulating is a theory refinement process. When refining a theory, an individual embarks on an iterative cycling procedure. We begin with a theory, code part of it, and soon detect gaps and fuzziness. Thus, we must return to theorizing before continuing to code. In other words, simulating involves both theorizing and coding. The investigator must address all possible variants that could occur. This is not done in a single programming session. Rather, a portion of a simulation is programmed as one moves back and forth between code and theory. Later, additional theoretical implications of these assumptions are realized. These then have to be programmed in. Generally, an individual goes through many cycles like the one described above. Thus, simulation programming is an iterative process requiring both theorizing and coding.

\section{A SAMPLE SIMULATION}

A brief description of a simulation that mimics the processes humans use while reading a text and that predicts recall of facts follows. Specifically, the process model builds two memorial representations of the textual material: a microstructure, or representation of the facts presented in a text, and a macrostructure, or representation of the main ideas derived from the text. This description is included to provide concrete examples for the next section, in which a description of the processes involved in simulating is provided.

\section{The Model: Data Structures}

The theory underlying the process model asserts that humans create multiple representations of a text in LTM. Specifically, a LTM macrostructure and a LTM microstructure are created. These memory structures are created during the encoding process. Individual segments of text are processed in STM and are then transferred to the LTM representations. In other words, the LTM. memory structures are appended as each chunk of text is processed in STM. These memory structures are hypothesized to be related. Each main idea or macroproposition is believed to point to a subtree or small portion of the overall micro- structure representation of the actual facts read. Specifically, the microstructure contains many subtrees, and each subtree contains facts or micropropositions related to a particular main idea or macroproposition. Thus, each main idea is associated with a subtree of the microstructure.

The facts contained in each subtree are hierarchically organized. Micropropositions that are more closely related to a specific main idea are located at higher levels of the hierarchical subtree than are those micropropositions that are less important to the main idea. Main ideas or macropropositions also vary in their importance to the text's overall theme. This variability in importance is represented by the level a macroproposition occupies in the macrostructure, or LTM structure of main ideas. The closer a macroproposition is to the top of the macrostructure hierarchy, the more important it is to the overall gist of the text. An example of a macrostructure and microstructure (including the relationship between subtrees in the microstructure and various macropropositions) can be seen in Figure 1.

\section{The Processes: Flow of Control}

The simulation created to represent how people process as text and form the memorial representations discussed above mimics the processes hypothesized to occur in reading. The following is the procedural flow of control implemented in the simulation.

(1) Determine a meaningful unit of text by using surface structure boundaries. Place this meaningful unit into an input chunk for further processing.

(2) If this is the first processing cycle, determine the topic of the text. Then connect the propositions in STM to make them coherent (see Appendix A). If not, connect the propositions or meaning units in STM to make them coherent and not just an unrelated set of facts. Appendix A describes the procedures for adding new propositions to STM in more detail.

(3) Transfer the items stored in the STM hierarchy to the LTM representation of the microstructure.

(4) If this is not the first processing cycle, search the input chunk for additional main ideas or macropropositions. If found, add to the LTM main idea representation (macrostructure) at a level that corresponds to the perceived importance of the main idea to the text as a whole. The procedures for adding new macropropositions to the macrostructure are presented in detail in Appendix B.

(5) Select items to remain in STM for additional processing cycles. Do this to create coherence between chunks of input information. Specifically, retain the last main idea added to the macrostructure to create local topic continuity and additional microproposition(s). Then empty STM of all items not selected for retention. The selection procedure for determining which items to maintain in STM is described in Appendix C.

(6) Recurse until the entire text is represented in LTM.

Specific examples are drawn from these in the next section, which describes the processes involved in designing and implementing a simulation. 


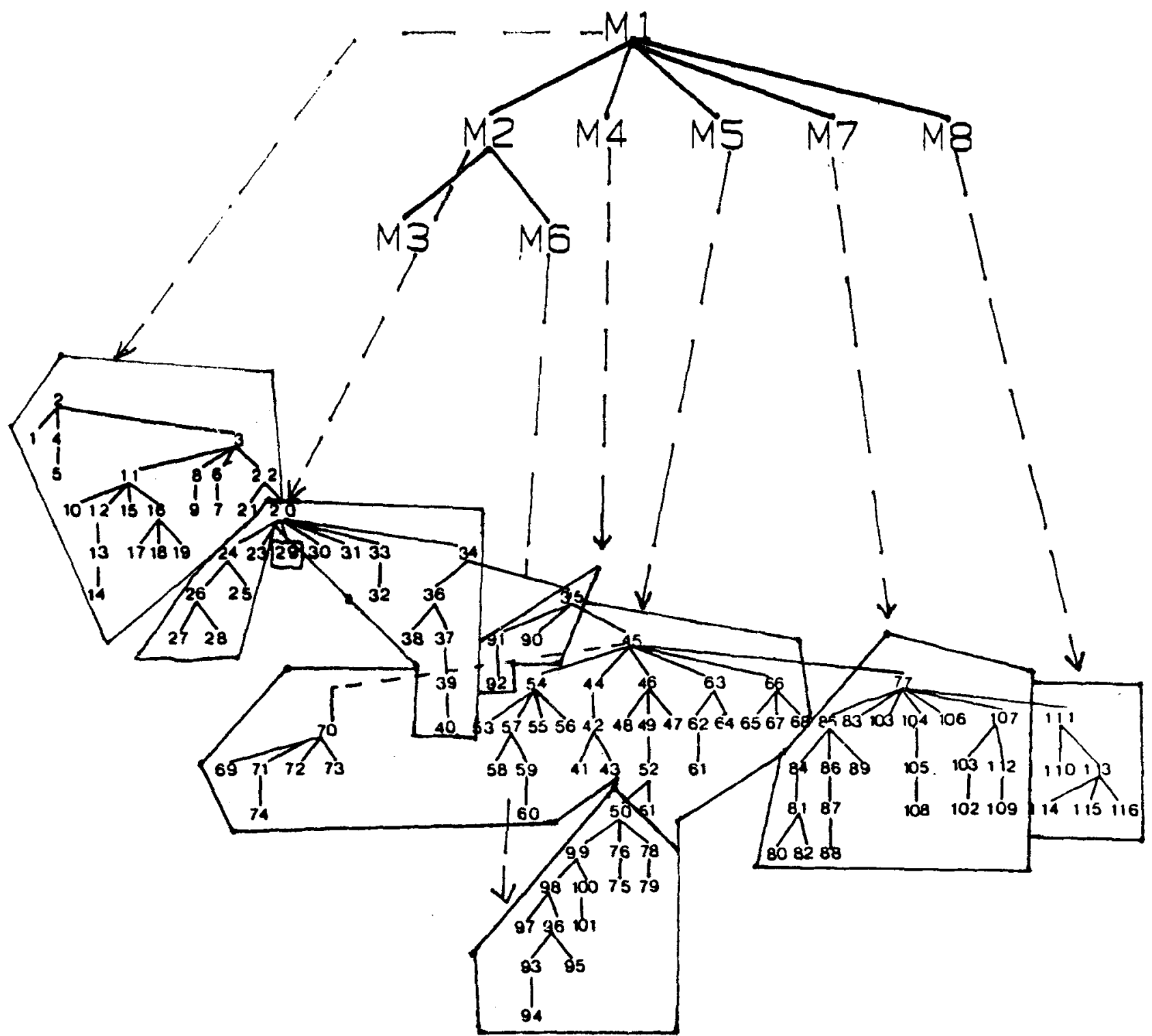

Figure 1. Example of macrostructure and microstructure, including relationship between subtrees and microstructure and various macropropositions.

\section{THE PROCESSES INVOLVED IN SIMULATING}

Three basic steps are necessary to create the example text processing simulation. First, it is necessary to isolate a manageable portion of the text processing domain. Second, an initial descriptive model of the theory is designed. Third, the theory is alternately coded and refined.

\section{Isolating the Problem}

The process of creating a simulation first requires the isolation of a manageable portion of a complex domain. The portion isolated must have the following two characteristics. First, it must be psychologically valid to view this portion in isolation. Second, the outcome of the simulation must yield testable predictions. Examples of the type of information that you want the simulation to yield include response latencies, predictions about the time required to conduct tasks with different kinds of processing requirements, recall probabilities, gaze durations, number of fixations, etc.

Problem isolation is one of the most difficult parts of the simulation task. In the example simulation, the domain of all memory and knowledge structures involved in text processing was initially chosen. This was later narrowed to the creation of a macrostructure and the rules that guide its formation. However, it became necessary to represent the microprocessing portion of the domain, 
because the two appeared to interact and it was deemed psychologically invalid to view macroprocessing in isolation.

On a first simulation, it is advisable to stay away from having semantic knowledge in the system; process models are much easier to deal with. Process models have all of the demands of creating a simulation without the added complexity and interaction of complex knowledge structures. Thus, it is best to limit initial simulations to modeling processes, production rules, etc. There is a need to theoretically justify looking at a particular process in isolation. Deciding just what to tackle is a demanding task.

\section{Creating an Initial Descriptive Model}

Following problem isolation, it is necessary to design an initial descriptive model. One begins by defining the structures and processes that are important to the domain chosen. For example, is it necessary to include both a STM component and a LTM component, or is only a STM component necessary? What types of processing are occurring in these memory structures, and when do they occur? In other words, after isolating the problem, create a global flowchart (or program outline) of what occurs, where it occurs, and how it operates.

While total a priori specification is impossible, the more concrete and specific the initial descriptive model, the easier the coding will be. Note that many of the processes that occur in the infamous "black boxes" will only become apparent while you are coding. However, it is necessary to know the purpose and general functions performed in the box.

The initial descriptive model for the example simulation was designed as follows. First, the general flow of control was mapped. The author knew that textual information had to be chunked into small sets of meaningful units (chunks of micropropositions). Then the chunks had to be input to STM for processing. In STM, the chunks of propositions needed to be checked for coherence, in order to relate the items to one another. Additionally, a search for main ideas had to be conducted in STM. Prior research had suggested that the items represented in STM are transferred to LTM representations. However, some items have to be retained in STM to assist in processing the next incoming chunk of micropropositions, so that individual chunks can be related.

Next, basic heuristics for connecting newly derived macropropositions with existing macropropositions in the LTM macrostructure were designed. These emphasized the relative importance of main ideas to the text topic, and therefore a hierarchical data structure was used to represent importance. Although this was a far cry from the amount of specification needed to completely code the simulation, it was enough to begin coding and embark on the cyclical coding-theory refinement process.

\section{Coding and Theory Refinement}

After choosing a limited domain and creating an initial process model, you are ready to embark on the coding- theory refinement cycle. To do this, take a small portion of the model, for example, one box in the flowchart, and derive rules that drive its operation. Then code these rules and the data structures they operate on. (It is usually best to put individual rules or groups of rules into separate functions, but I will address this problem later.) The main point is to begin to code small portions of a model and then embark on the process of iterative theory refinement. During coding, many situations that had not been considered previously are encountered. Remember, the manner in which these situations are resolved must be psychologically valid. ${ }^{3}$ The following paragraphs explain this refinement process.

In the example simulation, once it had been determined that macropropositions should be added to the macrostructure on the basis of importance, the following rule was coded:

If an incoming macroproposition overlaps with more than one existing macroproposition, it should be connected to the most important one it overlaps with.

During the coding process, it was realized that, although an overall rule had been coded, the possibility that a newly derived macroproposition could share common referents with two equally important macropropositions existed. Thus, it was decided that if this exception occurred, the program would determine which macroproposition the new macroproposition shared more common referents with. If this could be determined, the new macroproposition would become a child of the macroproposition it was most closely related to. Still more possibilities existed. For example, what if an incoming macroproposition was equally related to two macropropositions of equal importance. In this situation, the new macroproposition was connected to the most recent one. (This procedure is outlined in Appendix B.) However, three additional factors had to be dealt with. First, what if a new macroproposition did not overlap with any existing macroproposition? Second, was a psychologically plausible LTM retrieval mechanism that searched for importance implemented? Third, how could recency be judged in a psychologically plausible manner? This is just a small example of the type of work involved in coding a very well prespecified set of rules.

Thus, the process of implementation is as follows. First, rules are created and translated into code. Second, new pieces are recursively or cyclically considered and implemented in a psychologically plausible manner. The process continues until all parts of the problem area have been incorporated into the code. This is an inevitable part of designing and coding simulations. The specifics of searching, adding to a data structure, ordering rules, etc., are the types of items that have to be dealt with, and many may not have been previously considered in great depth. These have to be resolved within the constraints of prior research and psychological plausibility. In summary, once having defined a domain and its component processes, 
one begins the process of refining a theory while concurrently coding it. ${ }^{4}$

\section{Other Considerations}

There are two additional aspects of implementing a simulation. First, a simulation is by nature a generalpurpose algorithm for the chosen domain. The simulation allows one to derive predictions from diverse sets of data and situations. Thus, it is important to expound all possible occurrences or cases. Rules need to be created to handle the many possibilities that could hypothetically occur. The rule developed for dealing with a new macroproposition unrelated to all previous ones is an example of necessary expounding. (It can be found in the example of coding the rules for adding macropropositions to a macrostructure.) Although it is unlikely that any wellwritten text would introduce a main idea that is completely unrelated to previous input, it could happen. In fact, it often does in an essay produced by an unorganized writer. Thus, a general-purpose algorithm was created to address these possible inputs.

Second, the rules and procedures used in various sections of the code cannot be contradictory, or unintentionally interactive. Although one rarely intends to code contradictory rules, the cyclical coding process lends itself to such occurrences. By trying to maintain discrete functions in the theory/coding cycle, one can avoid some unintended interactions. It is easy to produce conflicting rules while fixing bugs or expanding the theory. A well-thoughtout inital design will avoid a lot of trouble later on.

\section{PROGRAMMING AND DEBUGGING NOTES}

\section{Making New Functions}

The beginning simulator should know how to compartmentalize (or modularize) code (i.e., which things should be made into separate functions?). Unless the modeler has experience with higher level languages (e.g., the $\mathrm{C}$ language), he/she is probably not accustomed to functions that call other functions that call yet other functions. Sequential function calls are vital to simulation programs. Code should be as compartmentalized as possible.

The basic rules to use when deciding what to make into functions are as follows. First, a single function should perform only one conceptual task. (For example, transfer items from STM to LTM.) Second, any code that is used many times should be a separate function. (For example, since micropropositions and macropropositions were continually being added to memory structures, separate functions were created to handle this task.) Third, rules and sets of related rules should be single functions ${ }^{5}$ (e.g., the rules for adding new macropropositions).

A heuristic for creating functions is:

Begin with a general flow graph. This graph should maintain the critical variables and call functions to perform operations on the input data. The functions should, in turn, call upon other functions to perform critical ac- tions. Finally, each function should perform only a single task.

Because the code is modular, one can easily test for correct data manipulation. Debugging is also simpler.

\section{Creating Local Variables}

Another issue is that prespecification of all local variables is usually not possible. Rather, you discover that an intermediate result needs to be stored. In these cases, a new variable should be created and added to the local variables that are declared inside the relevant function. Local variables are not needed when the intermediate results are not stored, that is, when the code manipulates only those variables initially passed to the function.

\section{Determining the Flow of Control}

Although global flowcharts assist the programmer, he/she needs to know precisely how items are stored and how they are manipulated within each function and between functions. Things do not always work as intended. It is not at all uncommon for something to occur during a test of a simulation on a variety of information such that one has no idea of how it occurred. For example, one may forget that portions of code have become obsolete. If control is passed to them, the programmer can be painfully reminded of their obsolescence. Similarly, he/she may discover conflicting rules for which no resolution mechanism has been provided because the rules were not thought to be conflicting. The latter is most likely to occur with revisions.

To minimize some of these puzzling and frustrating problems, begin by testing each function out of context, that is, as an individual unit. Examine the code line by line and statement by statement. See what is being stored in the variables and how the variables are being manipulated. Frequently, a lot of work is done in what can be referred to as "by-products" of functions. In other words, whereas a function returns a value (often $\mathrm{T}$ or $\mathrm{Nil}$ ), at the same time many data structures may become modified when procedures are implemented. The modified data structures are not returned by the function, but are most important to the process being simulated. By going through small sections of code, the modeler will have a much better idea of the precise flow of control, which assists during debugging.

\section{Debugging a Single Function}

The first step in debugging a complex simulation is to take each function out of context and test it on external, simple variables. For example, if the following function (written in INTERLISP)

(* This function adds a node to STM at level $\mathrm{N}$ in STM. It also adds the STM property level (N) to the node's property list)

\section{(DEFINEQ}

(ASSIGNLEVEL 


\section{(LAMBDA (PROP N) (PUTPROP PROP 'LEVEL-IN-STM N) (ADDPROP 'STM N PROP))))}

is included to place the property level-in-stm with the value $n$ onto the atom prop, and add the value prop to the property of STM called $n$, it is advisable to debug it by the following interactive procedure:

$\begin{array}{ll}\text { (SETQ PROP 'TEST-PROPOSITION) } & \left({ }^{*} \text { Initialize the variables) }\right. \\ \text { (SETQ N 10) } & \\ \text { (ASSIGNLEVEL) } & \left({ }^{*} \text { Call the function) }\right. \\ \text { (GETPROP PROP 'LEVEL-IN-STM) } & \left({ }^{*} \text { Get the property }\right. \\ & \text { value of the node) } \\ & \left({ }^{*} \text { Get the items at }\right. \\ \text { level N in STM })\end{array}$

In the above procedure, random values are assigned to the parameters passed to the function ASSIGNLEVEL. Next, the function is called and the results are evaluated. If the function works properly, the system should print " "10" as a response to (GETPROP PROP 'LEVEL-INSTM) and "TEST-PROPOSITION" to the test (GETPROP 'STM N) or (GETPROP 'STM 10). By using this method to step through each portion of the code contained in all the functions, the first debugging step can be easily completed. However, the individual functions must still be integrated and the integrated pieces tested as a whole.

\section{Printing}

Another debugging tip is to use many print statements in the early stages of debugging and to record all system output in a file. ${ }^{6}$ The exact procedure for recording a session will depend on the LISP dialect being used and the operating system. By recording a session and going through the output by hand, error checking is enhanced.

\section{Stepping Through Function Calls}

The most powerful way to debug is by breaking or stepping through functions at problem points in their execution. For example, if the function-by-function processes are operating correctly, but errors are made when the entire program is called, it is necessary to know why a parameter is not being passed properly or where rules are conflicting. Going through a printed output by hand can help narrow down the alternatives, but using well-placed breaks or step sequences can greatly reduce the confusion. The LISP language functions allow you to stop processing at particular stages and to query the program about the values and properties associated with various variables of interest.

\section{Stepping Through the Simulation}

Last, if the program should not work due to an error at execution time, it is most helpful to be able to move up and down the stack of functions executed to see exactly where the system encountered problems. This can be accomplished by using breaks. All system breaks allow you to query the system about values stored in variables, execute individual functions, print property lists of various atoms, etc. These are most useful in isolating a problem. In essence, LISP systems keep track of the functions they have executed and the values associated with local and global variables. Determining which function failed and the values associated with that function is very useful in determining where the code is failing and why.

\section{SUMMARY}

This manuscript was intended to provide the reader some insight into the process of creating a simulation of psychological processes. Of course, no brief description is a substitute for hands-on experience, even when the reader has an adequate background in the LISP progamming language.

Simulation programming is a cyclical, iterative exercise in theory refinement and coding. Problem domains need to be chosen carefully. Initial process models and flowcharts need to be as precise as possible. Most of the time spent on the project should be devoted to design. Theory refinement is necessary and leads to creating a general and powerful model for testing diverse sets of data. However, you only obtain meaningful results by scrupulously maintaining psychological validity in all portions of the code.

Creating cognitive simulations is a most rewarding experience. It is an intellectual exercise in both theorizing and programming. Creating a simulation not only provides the modeler the ability to empirically test predictions, it also buys the ability to generalize, because a simulation is a very general program that can deal with very diverse sets of data. In sum, interested readers should attempt hands-on simulation.

\section{NOTES}

1. Why LISP? Because LISP lends iself to modeling cognitive processes better than any other programming language. It is a symbolic programming language designed for recursion.

2. A prog is a primitive iteration mechanism in LISP. It iterates over local variables and allows labels, go statements, and return statements to be used.

3. It should be reemphasized that the need for iterative theory refinement is based on the fact that human cognitive processes tend to be ill defined.

4. For this reason, if one is not working alone, that is, if someone else is actually doing the coding in LISP, the theorizer must work very closely with the coder.

5. Especially if they are recursive.

6. In INTERLISP and COMMON LISP, this is easily accomplished by typing "DRIBBLE 'filename" before calling your top-level function and typing "DRIBBLE" after the program has finished executing. 
Appendix A

Procedure for Adding Micropropositions to STM

* Choose a critical microproposition for matching against input.

- Place all micropropositions in STM in a list.

* Order the list by level in STM and recency.

* Place Level 1 items at front of list.

* Sort Level 2 items, recency first.

* Place sorted Level 2 items after Level 1 items.

* Continue until the entire STM contents are represented in list.

- Choose critical micropropositions by taking the first item in the list.

* Assess all micropropositions in chunks for the presence of common referents, or common arguments held between the argument list of the critical microproposition and the micropropositions being assessed.

- Match against argument list Level 1 microproposition.

- Assess only argument lists of micropropositions in chunks.

- Assess micropropositions in order of primacy.

* When a microproposition in the chunk is found to share a common referent with the arguments of a critical microproposition:

- Attach a pointer between the micropropositions;

- Place the pointed-to microproposition at one level below that occupied by the critical microproposition it overlaps with.

* Make a new list of all micropropositions in STM that have not been used as the critical microproposition, choose a new critical microproposition, and recurse until the chunk has been emptied.

\section{Appendix B}

Algorithm for Adding New Macropropositions to the Macrostructure

The general heuristic is to conduct a top-down search for macropropositions contained in the macrostructure that share common referents with the new macroproposition. Thus, importance is emphasized over recency.

* Search for macropropositions that share common referents with the new macroproposition.

- Retrieve the Level 1 macroproposition and assess it for overlap with the new macroproposition.

- Retrieve all Level 2 macropropositions if the above fails.

- Continue assessing macropropositions at each level, until at least one overlapping macroproposition is found.

* Determine which macroproposition should point to the new macroproposition if more than one overlapping macroproposition is found at any one level in the macrostructure. If only one macroproposition is found, go to next step.

- Perform a secondary assessment to determine which macropropositions share the most common referents with the new macroproposition.

- If these are equal, select the most recent of the macropropositions.

* Add the new macroproposition to the macrostructure at Level $\mathrm{n}+1$, where $\mathrm{n}$ represents the level that the microproposition's parent occupies in the macrostructure.

* Place a pointer between the macroproposition selected by the above procedures and the new macroproposition.

\section{Appendix $\mathbf{C}$ \\ How Propositions are Selected for Additional Processing Cycles}

* Determine buffer size.

- Count the number of microproposition arguments in the last macroproposition added to the macrostructure.

- If this is Cycle 1, add 2 to the number obtained by counting microproposition arguments.

- If this is not Cycle 1 , add 1 to the number obtained by counting microproposition arguments.

* Maintain all micropropositions that are arguments of the last macroproposition added to the macrostructure in the buffer.

* Select additional micropropositions for holdover that were not arguments of the last macroproposition added to the macrostructure. 
- If a new macroproposition was added on this processing cycle, limit the choices to micropropositions added to STM during the current processing cycle.

- Select the microproposition(s) that are located at the highest level in STM that satisfy the above two considerations.

- If more than enough microcropositions can be located at high levels in STM, then choose the most recent one(s) for holdover.

* Add 1 to the cycle counters on all micropropositions held over in the buffer. 